\title{
El problema de Cauchy asociado a una perturbación no local de la ecuación de Benjamín-Ono periódica
}

\section{The cauchy problem associated to a non linear local perturbation of the periodic Benjamín-Ono equation}

\author{
Darwin Peña González ${ }^{1}$ \\ ${ }^{1}$ Magíster en Ciencias de la Matemática, Profesor Tiempo Completo, Universidad Autónoma del Caribe. \\ Email:darwindacier@hotmail.com.
}

Recibido 10/10/11, Aceptado 05/05/2012

\section{RESUMEN}

El propósito de este trabajo es estudiar el buen planteamiento local de la ecuación diferencial de Benjamin-Ono, $\partial_{t} u=-2 u \partial_{x} u-H \partial_{x}^{2} u$ la cual se le agregaran dos cantidades, una disipativa y otra de inestabilidad y mediante técnicas clásicas y en los espacios de Sobolev periódicos $\mathbb{H}^{\boldsymbol{s}}(\mathbb{T})$ mostraremos la buena colocación del problema de valor inicial, para $s>\frac{1}{2}$.

Palabras clave: Ecuaciones diferenciales parciales, buen planteamiento local del problema, espacios de Sobolev periódicos, Cauchy, Perturbación no local de la ecuación KdV.

\begin{abstract}
The purpose of this paper is to study the local sound approach to the differential equation of Benjamin-Ono $\partial_{t} u=-2 u \partial_{x} u-H \partial_{x}^{2} u$ which will add two numbers, one dissipative and one of instability and by classical techniques and periodic Sobolev spaces $\mathbb{H}^{s}(\mathbb{T})$ show good positioning of the initial value problem, for $s>\frac{1}{2}$.
\end{abstract}

Keywords: Partial differential equations, good local approach to the problem, periodic Sobolev spaces, Cuachy, No local perturbation of equation $\mathrm{Kdv}$.

\section{INTRODUCCIÓN}

La ecuación Korteweg-de Vries (KdV)

$\partial_{t} u t, x+u t, x \partial_{x} u t, x+\partial_{x}^{3} u t, x=0, t>0, x \in \mathbb{R}$

Ha sido objeto de intensas investigaciones en diversas áreas de la física y de la matemática tales como en la teoría de fluidos [1-5], sistemas completamente integrables [1], en ecuaciones diferenciales parciales [10] por mencionar algunas de ellas. Álvarez [2] estudia el problema de Cauchy asociado a una perturbación no local de la ecuación $(\mathrm{KdV})$. En este artículo se aborda el buen planteamiento local del problema de valor inicial

$$
\begin{gathered}
\partial_{t} u=\gamma \partial_{x}^{2} u-2 u \partial_{x} u+\mu H \partial_{x} u-\beta H \partial_{x}^{2} u \\
u t, 0=\phi t, \quad \phi \in C \quad 0, T ; H^{s}(\mathbb{T})
\end{gathered}
$$

En los espacios de Sobolev $H^{s}(T)$, donde $\Upsilon, \mu>0, \beta \in R$. La ecuación (2) es una perturbación disipativa de la ecuación

$$
\partial_{t} u=-2 u \partial_{x} u-H \partial_{x}^{2} u
$$

Donde (3) es la ecuación de Benjamin-Ono, la cual describe la interface entre dos fluidos de diferentes densidades y de profundidad infinita, en la que se le han agregado dos cantidades, una disipativa y otra de inestabilidad.

La ecuación (2) se origina en el estudio de fluidos y turbulencia del plasma [6]. Para esta propuesta, el buen planteamiento que haremos de (2), será en los espacios de Sobolev periódicos $\mathbb{H}^{\boldsymbol{s}}(\mathbb{T})$ y haciendo uso de técnicas clásicas probaremos aue (2) está localmente bien planteado en $\mathbb{H}^{s}(\mathbb{T})$ para $s>\frac{1}{2}$. 


\section{METODOLOGÍA}

Para la solución del problema (2) se realiza una consulta bibliográfica exhaustiva relacionada con las ecuaciones Korteweg-de Vries (KdV) y de Benjamin-Ono. Para probar la buena colocación del problema (2), se resuelve el caso lineal de la ecuación (2), luego probamos la equivalencia entre la ecuación diferencial e integral, para después mostrar la existencia, unicidad y por último la dependencia continua de la solución del problema (2) para el caso local.

\subsection{Elementos preliminares para la solución del problema planteado}

En esta sección presentaremos las notaciones y algunos lemas, proposiciones y teoremas que serán fundamentales.

1. H es la transformada de Hilbert de $f$ y es definida por

$$
\widehat{H f(k)}=-\frac{1}{2} \operatorname{sgn}(k) \hat{f}(k), \operatorname{con} \operatorname{sgn}(k)= \begin{cases}-1, & k<0 \\ 1, & k>0 \\ 0, & k=0\end{cases}
$$

2. Para $s \in \mathrm{R}$, el espacio $H^{s}(\mathbb{T})=\left\{f \in \mathrm{P}^{\prime}:\left(1+k^{2}\right)^{1 / 2}, \hat{f} \in \ell^{2}(\mathbb{Z})\right\}$ es el espacio Sobolev de orden el cual es un espacio de Hilbert con producto interno.

3. $(f, g)=\int_{-\infty}^{\infty}\left(1+k^{2}\right)^{s} \hat{f}(k) \bar{g}(k) d \xi$

Escribiremos

$$
\begin{aligned}
& A=-\left(-\mu H \delta_{x} u+\beta H \delta_{x} u+v d x u\right) \\
& B(k)=\frac{|k|}{2}+\beta \frac{i}{2} k|k|-k^{2} \\
& \left.E(t) \phi=\left(\mathrm{e}^{\mathrm{t}} \frac{|\mathrm{k}|}{2}+\beta \frac{\mathrm{i}}{2} k|k|-k^{2}\right) \phi(k)\right)^{v} \\
& \left.E(t) \phi=\left(\mathrm{e}^{\mathrm{t}(\mathrm{k})}\right) \hat{\phi}(k)\right)^{v}
\end{aligned}
$$

Lema 1: Sean $a, b \in[0+\infty]$ y $\lambda \geq 0$. Entonces existen constantes positivas y dependiendo solo de tal que

$$
c_{\lambda}\left(a^{\lambda}+b^{\lambda}\right) \leq(a+b)^{\lambda} \leq C_{\lambda}\left(a^{\lambda}+b^{\lambda}\right)
$$

Dms: Si $a=0$ no hay nada que probar. Asumamos que $a>$ 0 . Entonces, es equivalente a

$$
c_{\lambda}\left[1+\left(\frac{b}{a}\right)^{\lambda}\right] \leq\left[1+\frac{b}{a}\right]^{\lambda} \leq C_{\lambda}\left[1+\frac{b}{a}\right]^{\lambda}
$$

Así que es suficiente probar que hay constantes $c_{\lambda}$ y $C_{\lambda}$ tal que

$$
c_{\lambda}\left(1+r^{\lambda}\right) \leq(1+r)^{\lambda} \leq C_{\lambda}(1+r)^{\lambda}
$$

Para cualquier $r \in(0, \infty)$. Esto se sigue gracias a la función

$$
F(r)=\frac{(1+r)^{\lambda}}{1+r^{\lambda}}
$$

Teorema 1: (Del punto fijo de Banach). Si X es un espacio métrico completo y $A: X \rightarrow X$ es una contracción, es decir, existe $0 \leq C \leq 1$ tal que $d(A(x), A(y)) \leq C d(x, y)$ para todo par $x, y \in X$, entonces existe único $x A \in X$ tal que $A\left(x_{A}\right)=x_{A}$

Dms: Ver [5] y las referencias dadas allí.

Lema 2: Sean $\beta>0 . \gamma>0, \beta+\gamma>1, a \geq 0, b \geq 0$ y $g$ una función no negativa tal $t^{r-1} g(t)$ que es localmente integrable en $[0, T]$ suponga que

$$
g(t)=a+b \int_{0}^{t}(t-t)^{\beta-1} \tau^{\gamma-1} g(\tau) d \tau
$$

En $(0, T)$ entonces

$$
g(t) \leq a E_{\beta, \gamma}\left(b \Gamma(B)^{1 / 0} t\right)
$$

Donde $v=\beta+\gamma-1>0$

$$
E_{\beta, \gamma}(s)=\sum_{m=0}^{\infty} C_{m} s^{m v}
$$

Con $C_{o}=1$

$$
\frac{C_{m+1}}{C_{m}}=\frac{\Gamma(m v+\gamma)}{\Gamma(m v+\gamma)+\beta}
$$

Para todo $m \geq 1$

Dms: ver [3] y las referencias dadas alli

Teorema 2: Sean funciones medibles tales que

$$
\lim _{n \rightarrow \infty} f_{n(x)}=f(x)
$$

Para todo $x \in X$. Si existe $g \in L^{1}$ tal que para todo $x \in X$ y todo $n \in \mathbb{N}$ se tiene que $\mid f_{n(x)} \leq g(x)$, entonces $f \in L^{1} \mathrm{y}$

$$
\lim _{n \rightarrow \infty}\left(\int_{x} f_{n} d \mu\right)=\int_{x} f d \mu
$$

Dms: ver[14] y las referencias dadas allí

\section{Teoría local en $\mathbb{H}^{s}(\mathbb{T})$}

En esta sección mostraremos que el problema de valor inicial (2) en los espacios de Sobolev $\mathbb{H}^{s}(\mathbb{T})$, donde $v>0, \mu>$ $0, \beta \in \mathbb{R}$, está bien planteado localmente en $\mathbb{H}^{s}(\mathbb{T})$ para $s>\frac{1}{2}$. Para lograr este objetivo, probaremos que (2) es equivalente a la ecuación integral

$$
u t=e^{-t A} \phi-\int_{0}^{t} e^{-t-\tau A} \partial_{x} u^{2} \tau d \tau
$$

Donde A esta dado por (5) y $\phi \in \mathbb{H}^{s} \mathbb{T}$. Empezaremos nuestro estudio con la solución de la ecuación lineal asociada a (2) 


\section{La ecuación lineal}

Dedicaremos estaparte al estudio de la solución de la ecuación lineal asociada a (2), que por comodidad escribiremos

$$
\begin{gathered}
\partial_{t} u+A u=0 \\
u 0=\phi
\end{gathered}
$$

Donde A esta dado por (5) con

$$
v=1, \mu=1, \beta=1 \text { y } \phi \in \mathbb{H}^{s}(\mathbb{T})
$$

Teorema 3: (i)E: $[0, \infty] \rightarrow \mathfrak{B}(\mathbb{H} \mathbb{T})$ es un semigrupo en además,

$$
\|E(t)\|_{\mathfrak{B} \mathbb{H} \mathbb{T}} \leq\|\phi\|_{s}
$$

(ii) Sea $\lambda>0$ entonces $E(t) \in \mathfrak{B} \mathbb{H}^{s} \mathbb{T}, \mathbb{H}^{s+\lambda} \mathbb{T}$ para todo $t>0, s \in \mathbb{R}$ y satisface la desigualdad

$$
\|E(t) \phi\|_{s+\lambda} \leq C_{\lambda}\left(1+\left(\frac{\lambda}{t}\right)^{\frac{\lambda}{2}}\right)\|\phi\|_{s}
$$

Para toda $\phi \in \mathbb{H}^{s}(\mathbb{T})$, donde $C_{\lambda}$ es una constante que depende de $\lambda$

Proposición 1: La solución de (13) es $u(t)=E(t) \phi$

es decir, la aplicación $t \in[0, \infty] \rightarrow u(t)=E(t) \phi \in \mathbb{H}^{s}(\mathbb{T})$ es la única que satisface que

$$
\lim _{h \rightarrow 0}\left\|\frac{u(t+h)-u(t)}{h}-B u(t)\right\|_{s-2}=0
$$

Dms: Sean $\phi \varphi \in \mathbb{H}^{s}(\mathbb{T})$ y $u, v \in C\left(\left[0, \mathbb{T} ; \mathbb{H}^{s}(\mathbb{T})\right.\right.$ dos soluciones del problema (13) tal que $u(0)=\phi y v(0)=\varphi$. Entonces $w(t)=u(t)-v(t)$ satisface $(13)$ con dato inicial $w(0)=$ $\phi-\varphi$. De la prueba de (17) observe que

$$
\begin{gathered}
\left\|\frac{e^{-(t+h) A} \phi-e^{-t A} \phi}{h}-B e^{-t A} \phi\right\|_{s-2}^{2}=\left\|\left[\frac{e^{-h A}-1}{b}-B\right] e^{-t A} \phi\right\|_{s-2}^{2} \\
=\sum_{k=-\infty}^{\infty}\left(1+k^{2}\right)^{s-2}\left|\frac{e^{-A}-1}{b}-B\right|\|\phi\|^{2}
\end{gathered}
$$

Por el teorema del valor medio y el teorema de convergencia dominada (Teorema 2) nos permite obtener el resultado.

\section{Equivalencia de la ecuación diferencial e integral}

En esta sección probaremos que el problema (2) es locamente bien puesto en los espacios de Sobolev $\mathbb{H}^{S}(\mathbb{T})$.
Teorema 4: Si $s>\frac{1}{2}$, el problema (2) es equivalente a la ecuación integral

$$
u(t)=e^{-t A} \phi-\int_{0}^{t} e^{-(t-\tau) A}\left(\partial_{x} u^{2}(\tau)\right) d \tau
$$

Más precisamente, si $u \in C\left([0, T] ; \mathbb{H}^{s}(\mathbb{T})\right.$ con $s>\frac{1}{2}$, es una solución de (2) entonces satisface (18). Reciprocamente, si $u \in C\left([0, T] ; \mathbb{H}^{s}(\mathbb{T})\right.$ con $s>\frac{1}{2}$, es una solución de (18) entonces $u \in C^{1}\left([0, T] ; \mathbb{H}^{s-1}(\mathbb{T})\right.$ y satisface (2) con derivada dada por

$\lim _{h \rightarrow c}\left\|\frac{u(t+) h-u(t)}{h}-B u(t)+\left(\partial_{x} u^{2}(t)\right)\right\|_{s-2}=0$

Dms: La primera parte de la prueba es consecuencia del método de variación de parametros y de observar que el término no lineal $\delta_{x} u^{2}$ tiene sentido, pues puede considerarse como una distribución periodica, ya que $u^{2}$ es continua y se anula en el infinito para $s>\frac{1}{2}$, en virtud del lema de Sovolev. La segunda parte se obtiene reemplezando la de la ecuación (18) en (19) y por el teorema de convergencia dominada (Teorema 1), la continuidad fuerte $e^{t A}$ del grupo y el teorema del valor medio obtenemos el resultado.

\section{La existencia de la solución del problema (2)}

Teorema 5: Sea $\phi \in \mathbb{H}^{s}(\mathbb{T})$, con $s>\frac{1}{2}$. Entonces, existe $T=$ $T\left(\|\phi\|_{s}\right)>0$ y una única solución $u \in C\left([0, T] ; \mathbb{H}^{s}(\mathbb{T})\right)$.

Dms: La idea de la prueba es aplicar el teorema de contracción de Banach (Teorema 1) a la función definida por el miembro de (18) en el espacio adecuado. Con este fin definimos lo siguiente

$$
\Phi_{\mathrm{T}}(\mathrm{M})=\left\{u \in C\left([0, T] ; \mathbb{H}^{s}(\mathbb{T})\right):\left\|u(t)-e^{-t A}\right\|_{s} \leq M\right\}(20)
$$

El cual es cerrado en $C\left([0, T] ; \mathbb{H}^{s}(\mathbb{T})\right)$ y por tanto es completo. Luego existe $T>0$ tal que

$$
\begin{aligned}
& \Psi: \Phi_{\mathrm{T}}(\mathrm{M}) \rightarrow \Phi_{\mathrm{T}}(\mathrm{M}) \\
& u \mapsto \Psi(u)
\end{aligned}
$$

Y se muestsra que $\psi(u) \in C\left([0, T] ; \mathbb{H}^{s}(\mathbb{T})\right.$ es decir

$$
\left\|\Psi(u(t))-\Psi\left(u\left(t^{\prime}\right)\right)\right\|_{s} \rightarrow 0, \quad \mathrm{t}^{\prime} \rightarrow \mathrm{t}(21)
$$

Con $t \in[0, T]$. Luego $\psi$ es una contracción (Teorema 1), con loque existe un punto fijo.

\section{Buen planteamiento local en $\mathbb{H}^{s}(\mathbb{T})$ para $s>\frac{1}{2}$.}

Teorema 6: El problema (2) es localmente bien puesto en $\mathbb{H}^{s}(\mathbb{T})$ para $s>\frac{1}{2}$. Más precisamente, para $\phi \in \mathbb{H}^{s}(\mathbb{T})$, existe $T>0$ y una única solución $u \in C\left([0, T] ; \mathbb{H}^{s}(\mathbb{T})\right.$ que 
satisface (2) y tal que $u \in C\left([0, T] ; \mathbb{H}^{s-1}(\mathbb{T})\right)$. Además, la aplicación de $\phi \in \mathbb{H}^{s}(\mathbb{T}) \mapsto u \in C \quad 0, T ; \mathbb{H}^{s}(\mathbb{T})$ es continua en el siguiente sentido: Sean $\phi_{n} \in \mathbb{H}^{s}(\mathbb{T}), n=1,2, \ldots$, tales que $\phi_{n} \rightarrow \phi$ y sean $u_{n} \in C\left(\left[0, T_{n}\right] ; \mathbb{H}^{s}(\mathbb{T})\right)$ soluciones de (2) con $u_{n}(0)=\phi_{n}$. Entonces, las soluciones pueden ser extendidas si es necesario al intervalo $[0, T]$ para $n$ suficientemente grande y

$$
\lim _{n \rightarrow \infty} \sup _{[0, T]}\left\|u(t)-u_{n}(t)\right\|_{s}=0(22)
$$

Dms: Supongamos que $v \in C\left([0, T] ; \mathbb{H}^{s}(\mathbb{T})\right), v(0)=\psi \in \mathbb{H}^{s}$ (T) con $s>\frac{1}{2}$ es solución de (2) con dato inicial $\psi$. Entonces

$$
\begin{gathered}
\|u(t)-v(t)\|_{s} \leq e^{-t A}(\phi-\psi)\left\|_{s}-\int_{0}^{t}\right\| e^{-(t-\tau) A}\left(\partial_{x}\left(u^{2}-v^{2}\right)\right)(\tau) \|_{(s-1)+1} d \tau \\
\leq\|\phi-\psi\|_{s}+\int_{0}^{t}\left(1+\frac{1}{\sqrt{t-\tau}}\right)\left\|\partial_{x}\left(u^{2}-v^{2}\right)\right\|(\tau)_{s-1} d \tau \\
\leq\|\phi-\psi\|_{s}+\int_{0}^{t}\left(1+\frac{1}{\sqrt{t-\tau}}\right)\left\|u^{2}-v^{2}\right\|_{s} d \tau \\
\leq\|\phi-\psi\|_{s}+\int_{0}^{t}\left(1+\frac{1}{\sqrt{t-\tau}}\right)\|(u+v)(u-v)\|_{s} d \tau \\
\leq\|\phi-\psi\|_{s}+\int_{0}^{t}\left(1+\frac{1}{\sqrt{t-\tau}}\right)\|u+v\|\|u-v\|_{s} d \tau \\
\leq\|\phi-\psi\|_{s}+\int_{0}^{t}\left(1+\frac{1}{\sqrt{t-\tau}}\right)\left(\|u\|_{s}+\|v\|_{s}\right)\|u-v\|_{s} d \tau \\
\leq\|\phi-\psi\|_{s}+\left(\|u\|_{s, \infty}+\|v\|_{s, \infty}\right) \int_{0}^{t}\left(1+\frac{1}{\sqrt{t-\tau}}\right)\|u-v\|_{s} d \tau \\
\leq\|\phi-\psi\|_{s}+K_{s} \int_{n}^{t}\left(\frac{1}{\sqrt{t-\tau}}\right)\|u-v\|_{s} d \tau
\end{gathered}
$$

Por el lema 2

$$
\|u(t)-v(t)\|_{s} \leq\|\phi-\psi\|_{s} E\left(\left(K_{s} \Gamma\left(\frac{1}{2}\right)^{2}\right) t\right)
$$

Con

$$
\boldsymbol{E}(s)=\sum_{m=0}^{\infty} c_{m} s^{\frac{m}{2}}
$$

Con lo que converge uniformente en $[0, T]$ y es creciente dado que,

$$
\frac{c_{m+1}}{C_{m}}=\frac{\Gamma\left(\frac{m}{2}+1\right)}{\Gamma\left(\frac{m}{2}+\frac{1}{2}+1\right)}
$$

Donde $\Gamma$ es la función Gamma. Por consiguiente

$$
\|u(t)-v(t)\|_{s} \leq\|\phi-\psi\|_{s} E\left(\left(K_{S} \Gamma\left(\frac{1}{2}\right)^{2}\right) T\right)
$$

Lo cual muestra la dependencia continua. Y si $\psi=\phi$ entonces $u=v$ con lo que se muestra que es única.

\section{CONCLUSIÓN}

- Se demostró que el problema (2) está bien puesto en los espacios de Sobolev periódicos con dato inicial, para es decir, se mostro la existencia la unicidad y la dependencia continua de (2).

\section{REFERENCIAS}

[1] Bao-Feng Feng, T. Kawahara, Multi-hump stationary waves for a Korteweg-de Vries equation with nonlocal perturbations, Physica D, 137, (2000), pp. 237-246.

[2] Borys Y. Alvarez S., On the Cauchy problem for a nonlocal perturbation of the KdV equation, Tesis Doctoral, IMPA, 2002.

[3] Henry, Geometric theory of semilinear parabolic equation, Lectures Notes in Mathematics, vol. 840, Springer (1957).

[4] N. Alon, R. A. Duke, H. Lefmann, V. Rodl, and R. Yuster. The algorithmic aspects of the regularity lemma. J. Algorithms, 16(1): 80-109, 1994.

[5] PrusakR. J. Iorio, Jr., Valeria de Magalhaes Iorio, Fourier Analysis and Partial Diferential Equations, Cambridge studies in avanced mathematics, 70, (2001).

[6] Qian, H.H. Chen, and Y.C. Lee, A turbulence model whit stochastic soliton motion, Departemento Physics and astronomy. The laboratory for plasma and fusion energy studies, University of Maryland,College Park, Maryland 20742. (Received 19 may 1988; accepted for publication 20 september 1989).

[7] R. J. Iorio, Jr.,On the Cauchy porblem for the BenjaminOno Equation, Comm. PDE, 11, (1986), pp.1031-1081.

[8] R. J. Iorio, Jr.,The Benjamin-Ono Equation in Weighted Sobolev Spaces, J. Math. Anal. Appl., Vol.157, No. 2, (1991), pp. 577-590.

[9] R. J. Iorio, Jr.,KdV, BO and Friends in Weighted Sobolev Spaces, Function Analytic Methods for Partial Diferential Equations, Springer-Verlag, vol. 1450 (1990) pp. 105-121.

[10] T. Kato, Nonstationary Flows of Viscous and Ideal Fluids in R3, Journal of functional Analysis, 9 (1972), pp. 296-305.

[11] T. Kato, On the Cauchy problem for the (Generalized) Korteweg-de Vries Equation, Studies in Applied Mathematics, Advances in Mathematics Supplementary Studies, Vol. 8,(1983), pp. 93-128. 
[12] T. Kato and H. Fujita, On the non-stationary NavierStokes system, Rend. Sem. Mat. Univ. Padova, 32(1962), pp.243-260.

Regularity lemma. J. Algorithms, 16(1): 80-109, 1994.
[13] T. Roger. In_nite-dimensional dynamical systems in mechanics and physics. Springer-Verlag. p(50), 1988.

[14] W. Rudin. Real and Complex Analysis. McGraw-Hill. $\mathrm{p}(28), 1970$. 